\title{
A design oriented multiaxial stress-based criterion for the strength assessment of adhesive layers
}

\author{
A. Spaggiari*, D. Castagnetti, E. Dragoni \\ Università degli Studi di Modena e Reggio Emilia - Dipartimento di Scienze e Metodi dell'Ingegneria, Via G. Amendola 2, 42122, Reggio Emilia, Italy
}

\section{ART ICLE INFO}

\section{Keywords:}

Failure criterion

Stress analyses

Adhesive

Experimental tests

Multiaxial stress

\begin{abstract}
A B S T R A C T
Adhesively bonded joints are becoming widespread in the composites industry and therefore there is a need for quantitative information on the mechanical strength of the material used. The great strength and stiffness of a composites structure may be strongly undermined by their weakest part, the bonded joint. Unfortunately, the testing of adhesives in bulk form may not be representative of their behaviour in a layered state, typically quite thin, because of differences in the polymerization process and lack of adhesive-adherend interfaces. The drawback of the test in thin layer is the stress concentration at the edges, typical in the single lap or t-peel joints, and also the chance of having the adhesive subjected both to a shear and predominant peel stress. This work deals with the characterization of adhesives in thin film under uniform distributions of multi-axial stresses, which is the typical application condition. The test exploits a tubular butt-bonded specimen, previously investigated by the authors, which guarantees a non-singular stress field over the adhesive layer both in shear and normal directions. According to the analytical prediction, in addition to the direct normal stress, both radial and circumferential secondary stresses arise in the adhesive, due to the constrained lateral contraction imposed by the adherends (Poisson's effect). The test campaign investigates two chemically different, commercial adhesives, an acrylic and an epoxy resin. By means of a biaxial testing machine, we applied to the specimens eight different combinations of normal and shear loads ranging from pure tensile to a shear-compressive stress state. As expected, both the pure shear stress and the compressive stresses lead to better performances of the adhesive layer with respect to tensile loading. The authors compare a variety of failure criteria from the literature and propose a simple multiaxial criterion to obtain a failure envelop of the experimental data. The applicability of the criterion is also assessed on experimental tests found in literature on different configurations and gives fairly good results. The outcome of study is a simple stress based, failure criterion, which can be used to predict the failure of several adhesive bonded joints, relying only on monoaxial experimental data.
\end{abstract}

\section{Introduction}

The present work deals with the quasi static characterization of a thin layer of structural adhesives through an ad-hoc tubular joint previously developed by the authors. The motivation of the work comes from the need of reliable and simple tools to design bonded structures in an industrial context. Normally in technical literature, two completely different approaches are envisioned. The first one exploits bulk specimens, made with the adhesive with standard dog bone shapes and tested on a tensile machine in the same manner as for metallic materials [1], [2]. The second one determines the adhesive properties by using it in thin film, as typically applied in real applications due to their superior performances [3], [4]. Adhesive producers typically recom- mend a quite thin adhesive layer for high performance structural joints, according to Huntsman "A layer of adhesive $0.05-0.10 \mathrm{~mm}$ thick will normally impart the greatest lap shear strength to the joint" [5] while according to Henkel Loctite the high performance, high stiffness joints are obtained with thin adhesives [6]. Well-established specimens typically possess the single, double or other lap specimen configurations, like the ASTM Standards D1002 and ASTM D3528. However, although simple to make and test, these geometries have complex stress distributions within the bond and give rise to both normal and shear stresses that vary from point to point [7], [8]. Stress singularities at re-entrant corners and at points of material discontinuity are also an issue [9] that undermine the meaning of these tests for providing genuine strength properties of the adhesive [10].

\footnotetext{
* Corresponding author.

Email address: andrea.spaggiari@unimore.it (A. Spaggiari)
} 


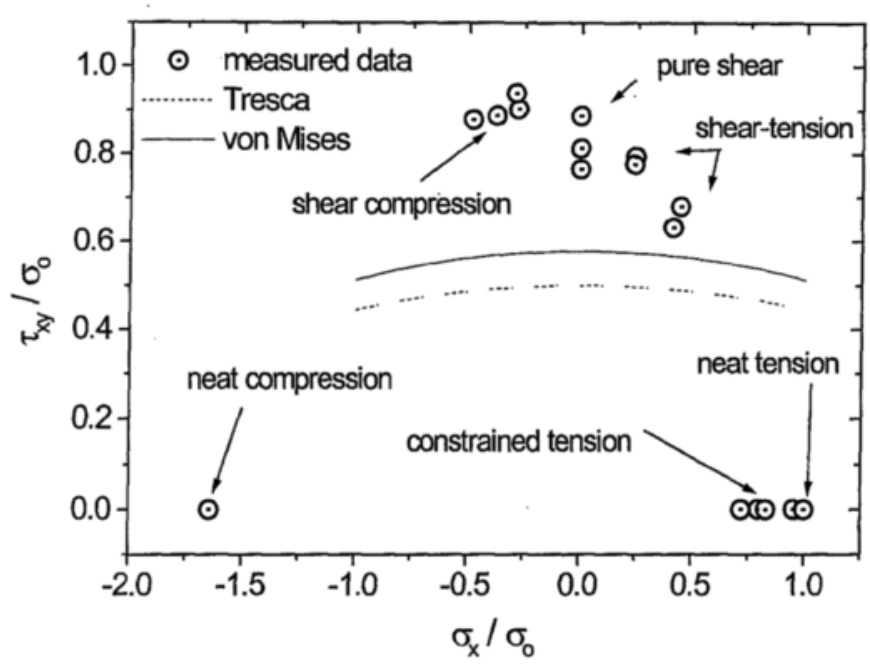

Fig. 1. Experimental data from Ref. [16] on highly constrained adhesive. Classic von Mises and Tresca criteria fails at creating a failure envelop region, since they do not consider the stress triaxiality.

Both these procedures have advantages and drawbacks. On one hand, the bulk test does not depend on the specimen geometry, the failure strain and stresses are very easy to calculate and the surface preparation of the adherends is not an issue. However, a possible drawback is the potential presence of defects and porosity in the bulk adhesive, not typical in real thin film applications and the complexity of using the failure stress of the bulk for the adhesive joint design, [11]. Even though there has been research work performed on how to avoid defects and porosity during the manufacturing process [12] the difference between thin film and bulk still can be a potential issue [13]. This problem is due to the constant presence of very rigid adherends, which create strong stress concentrations at the corners and highly triaxial stress state in the polymer. The high stress triaxiality is detrimental for the adhesive strength, since, as for many polymers, the adhesives are quite sensible to the mean positive stress applied [7] [8], both for ductile and brittle adhesives. Since the aim of the research is to propose a simple criterion applicable even in the industrial world, the authors consider the adhesive as a two-state material: structurally intact whilst in the elastic linear region, failed in case of plasticization and damage. Although one may argue that this is an oversimplification which can be too conservative, in many situations it is essential that the plasticity of the structural member is to be avoided and therefore this statement is applicable to many real-life situations. The first idea, which is the most natural one, is to borrow from the metal world the standard "von Mises criterion". Some efforts have been made in technical literature [16], but soon the need of an alternative criterion to the classical "von Mises ideal" stress came out, as shown in Fig. 1. The motivation is that the von Mises criterion considers only the distortion strain energy and disregards the dilation strain energy. The main advantage of the thin film test for the adhesive characterization is that the experimental properties are retrieved in a condition that is quite similar to the real application, with the adhesive constrained between two rigid adherends and with the typical thickness used in industrial context.

Two drawbacks are envisioned when testing adhesives in thin film. First, the properties depend on the adherends' material type (steel, aluminium) and on the surface preparations [9] [10], while the adhesive strength is at least function of its thickness [11-13]. Second, considering the typical specimen recommended by ASTM standards, such as the single or double lap joint or the Double Cantilever Beam, it is easy to understand that severe stress concentrations occur at the corners. This phenomenon comes from the elastic mismatch between stiff adherends and flexible adhesive and perturbs the measure of the adhesive properties in the elastic and post-elastic range. These stress concentrations, which can lead to singularities in the stress field, are considered an important dangerous trigger for cracks. Every joint has a different intensity of the stress concentration, according to Goglio et al. [21] [22],

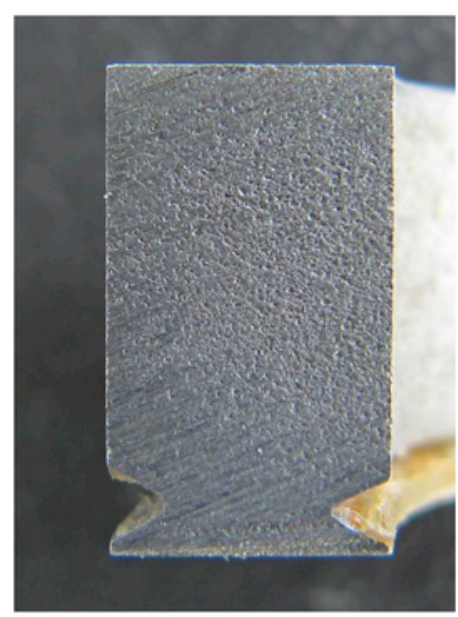

(a)

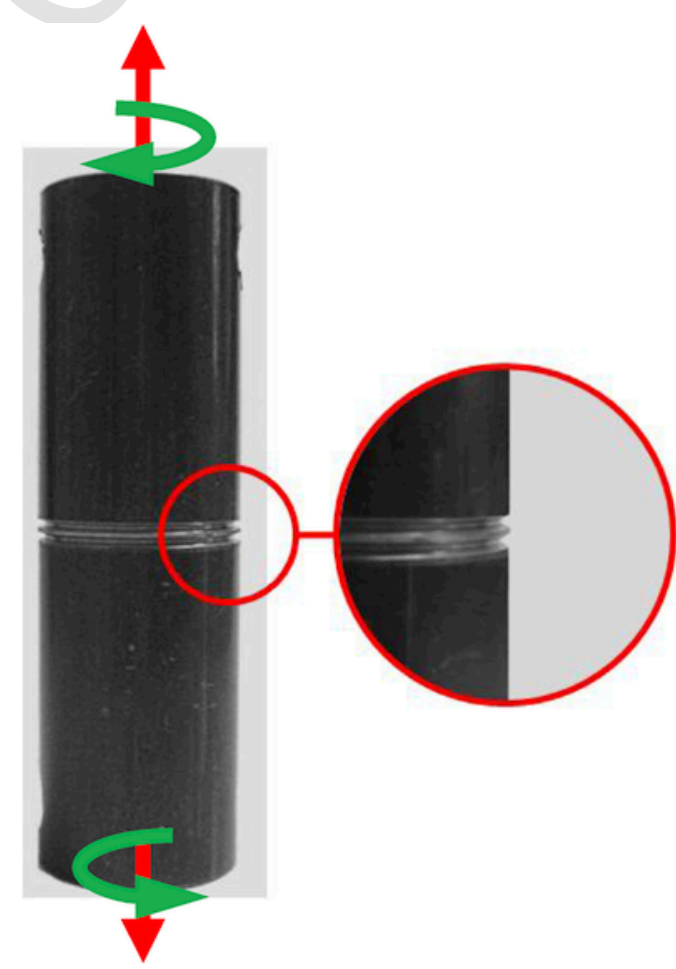

(b)

Fig. 2. Modified butt joint (b) proposed by the authors, and magnified image of the internal and external groove (a). 


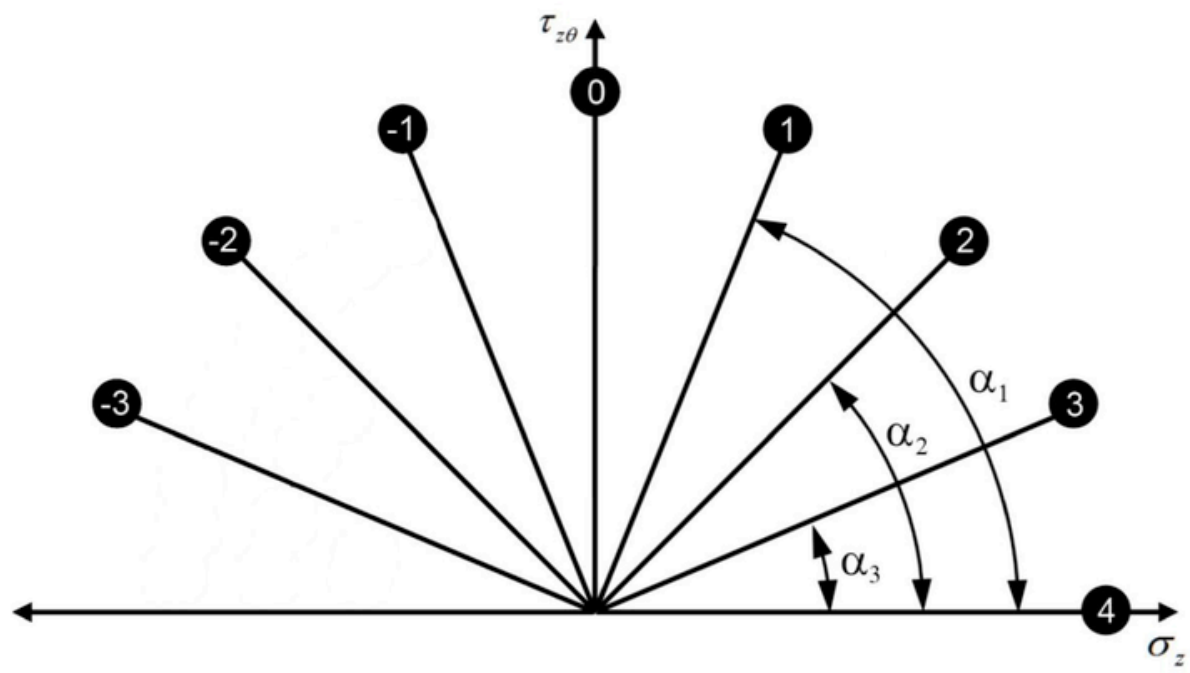

Fig. 3. Configurations tested in the sigma-tau plane.

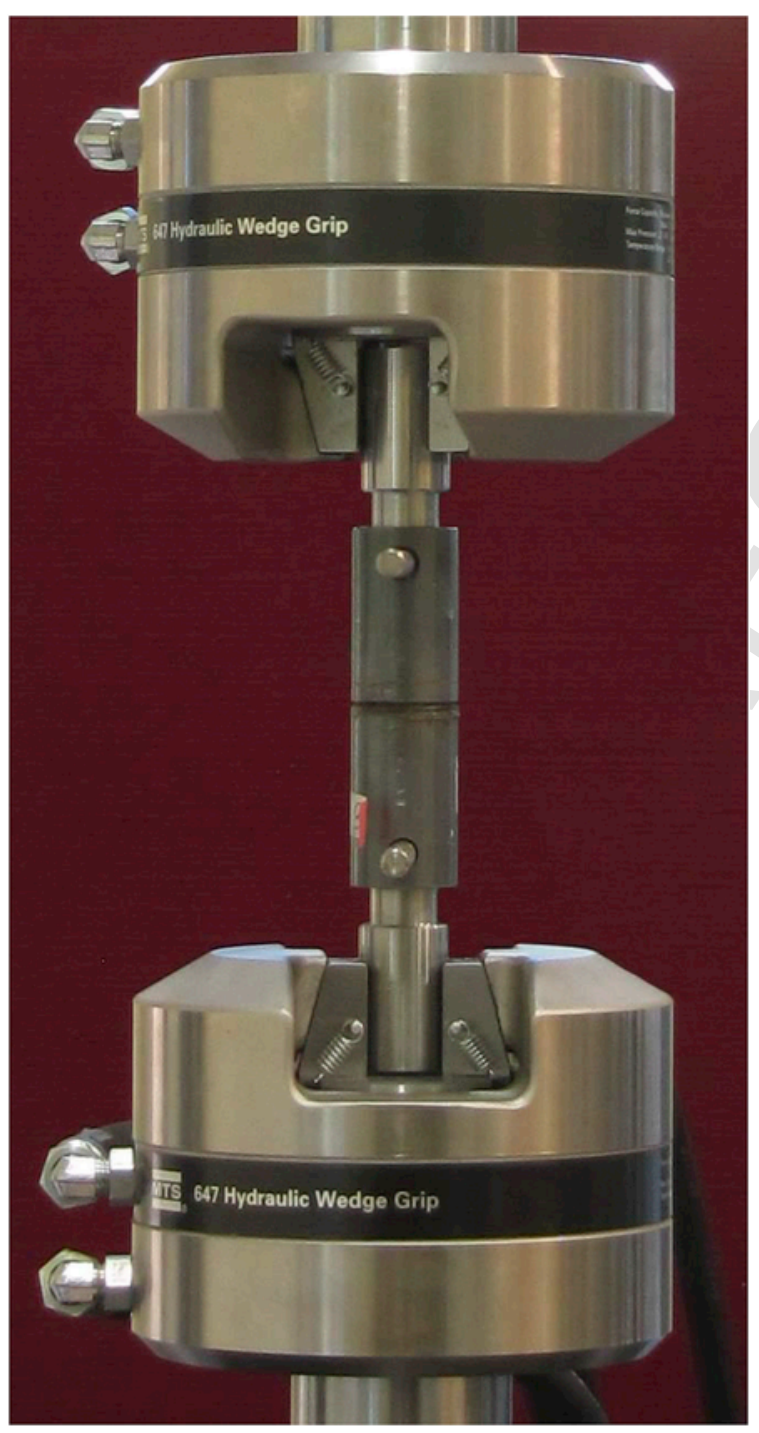

Fig. 4. Experimental set up on the biaxial test machine MTS Bionix 858 . which causes complex problems when comparing adhesive properties retrieved with different specimens. Moreover, it would be important to understand the failure stresses both in tension (mode I) and shear (mode II) [23], since the adhesive behaviour is quite sensitive to the loading direction. Unlike the metals, the polymers are stronger, tougher and more ductile in mode II, while the failure stress and the fracture toughness are lower in mode I. Recently, several authors tried to exploit different specimen geometries in order to apply different loading modes on the same specimen. One interesting configuration involves butt-bonded adherends, so called "Napkin ring" test [24], and this shape has been proven useful both in torsion [19] and in tension [25] by adding stress relief grooves which are able to lower the stress concentrations at the adhesive interface and in the bond line, see Fig. 2. This architecture has two advantages; first, the machining of the adherends is very simple, especially compared to the ASTM Arcan and TAST (ASTM D5656) tests, which has the same purpose. Specifically, the proposed geometry requires a simple turning of the tubular material with a threading tool; second, this configuration allows any desired combination of tension/ compression and torsion to be easily obtained. The only additional constraint is that the ASTM tests exploit a simple uniaxial test machine, while the tubular one needs a biaxial machine capable to apply tension and torsion at the same time. Other possible mixed mode test specimen configurations are the mixed mode double cantilever beam [26], the single leg bending [27] and the mixed mode fracture [28] and the modified Arcan test [29] which need quite complex equipment and could hide some potential problems as already discussed in [30].

The present paper exploits the modified butt joint with relief grooves to characterize two different adhesives in thin film. The first one is the Loctite Multibond 330, a general purpose acrylic adhesive [31], while the second one is the Loctite Hysol 3422, a two component, fast curing, epoxy resin [32].

The two adhesives were tested and the failure loads and torques are registered. Thanks to the simple geometry adopted and the absence of stress concentrations, an analytical stress prediction is possible and therefore the maximum normal stresses and shear stresses are calculated. 


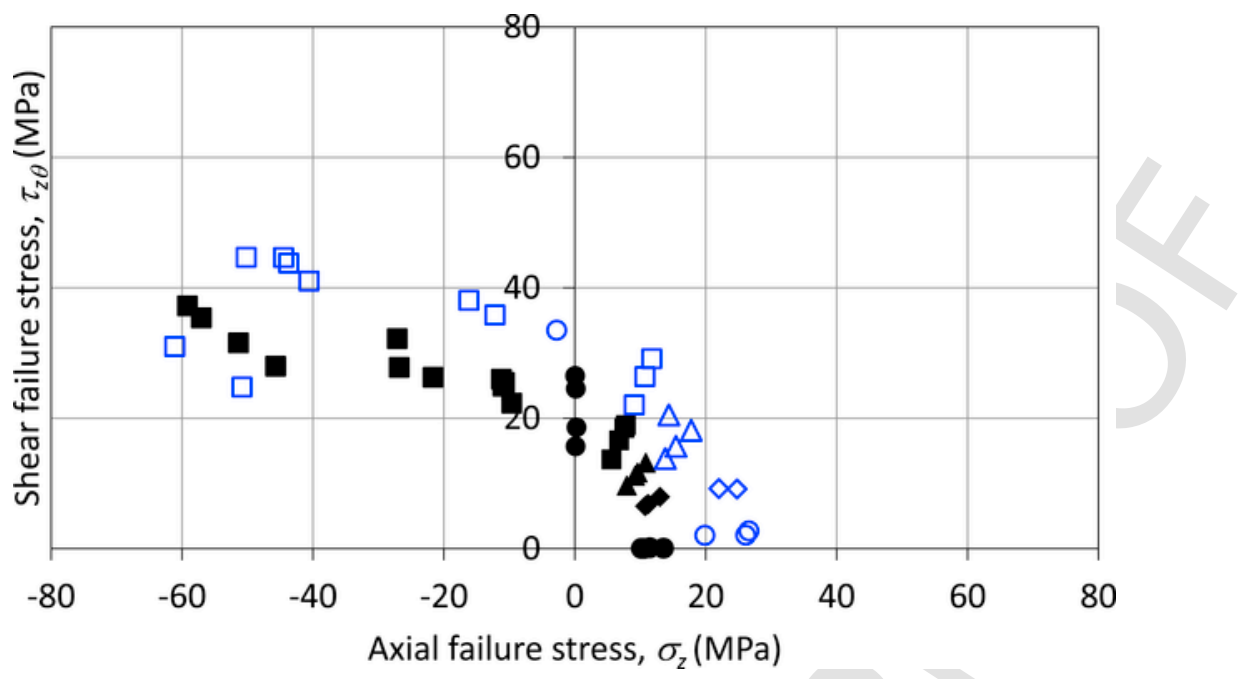

Fig. 5. Shear, $\tau_{z \theta}$, versus axial, $\sigma_{z}$, failure stresses retrieved from the experimental tests: Loctite Multibond 330 (solid symbols), Loctite Hysol 3422 (empty symbols).

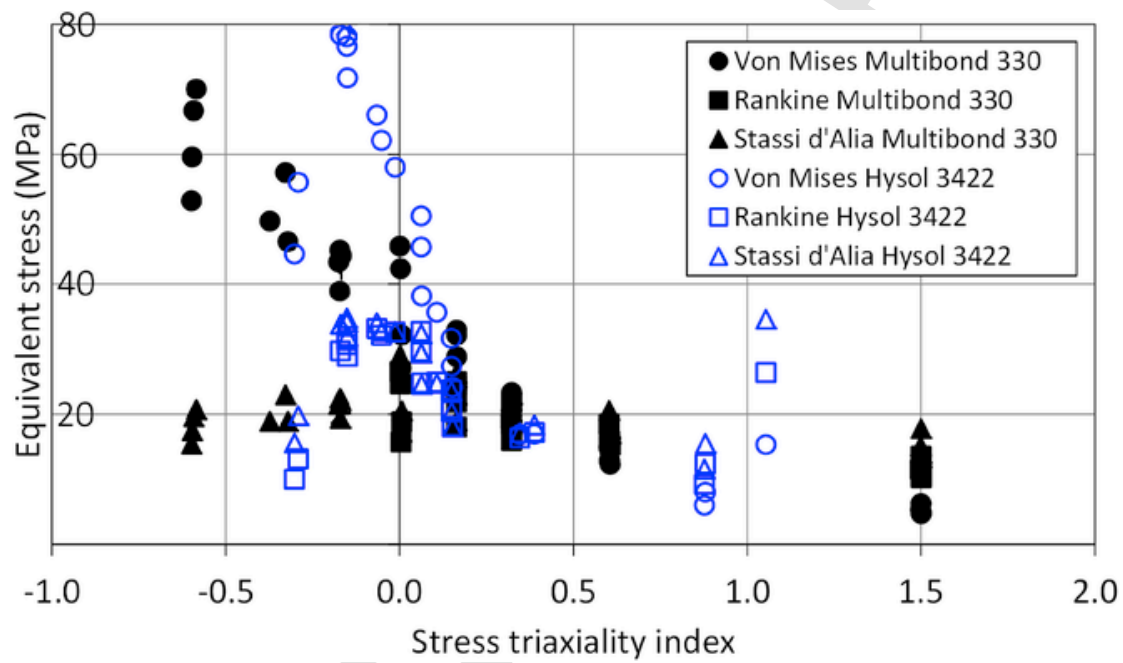

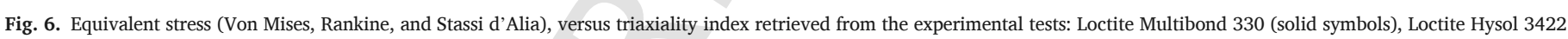
(empty symbols).

Table 1

Parameters of the proposed failure criterion.

\begin{tabular}{llll}
\hline Adhesive & $\tau_{c r}[\mathrm{MPa}]$ & $\sigma_{a v}[\mathrm{MPa}]$ & $A[\mathrm{MPa}]$ \\
\hline Loctite Multibond 330 & 21.3 & 11.5 & 39.5 \\
Loctite Hysol 3422 & 33.5 & 24.2 & 46.3 \\
\hline
\end{tabular}

\section{Materials and method}

\subsection{Experimental campaign}

We decided to design the experimental campaign according to the "Design of Experiment" approach [33], where the input variables considered are the axial loading and the shear loading applied to the joint. By combining the two loading conditions, a systematic investigation of the axial $(\sigma)$ - shear $(\tau)$ plane is carried out. The experimental loading conditions involve pure tensile, pure shear and six configurations of mixed loading both in tension and in compression. It was not possible to test the thin adhesive in pure compression since it would have been very difficult to identify the failure point. In fact, having a very thin adhesive lead to possible contact of the adherends, which has to be avoided. Moreover the adhesive properties in compression are better than in tension since polymers are typically quite sensitive to stresses triaxiality [34] so the performed test are more conservative.

Fig. 3 illustrates the eight configurations tested. The positive numbers identify a tensile loading condition, while the negative ones a compressive loading condition. The pure shear test is labelled with a zero.

To define quantitatively these eight experimental loading conditions, we used the angle $\alpha$ of the line in the $\sigma-\tau$ plane, defined as:

$\alpha=\operatorname{arctg}\left(\frac{\tau_{z \theta}}{\sigma_{z}}\right)$

The tubular specimens where manufactured in mild steel (Fe 360) with a Young Modulus of $206000 \mathrm{MPa}$ and a Poisson's ratio of 0.33 . The adherends where prepared according to a standard internal procedure which ensures a good trade-off between proper bonding and ease of manufacturing. First, the adherends were cleansed by a specific product, the Loctite 7030, to remove any greasy substances which can lubricate the surface preventing a proper bonding. According to ASTM specifications and technical literature [17] [35] [36], we continued the surface preparation of the adherends by sandpapering (with P200 foil) and cleaning again with Loctite 7030. Caution must be paid when ap- 


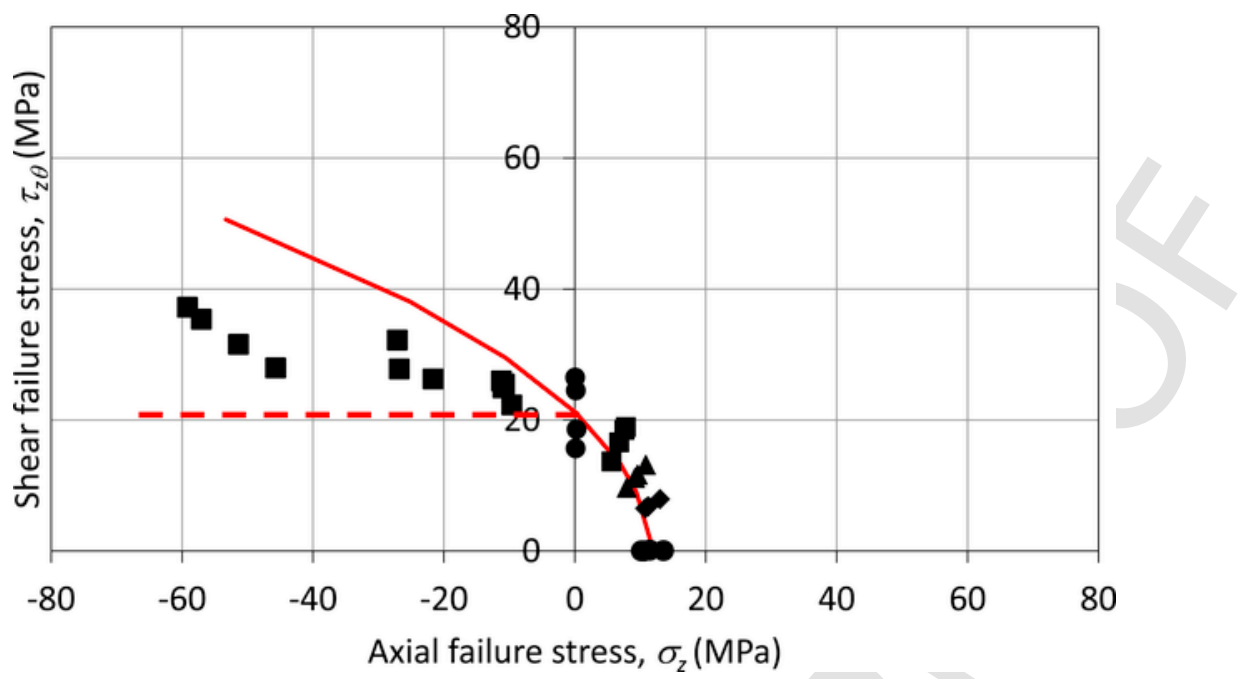

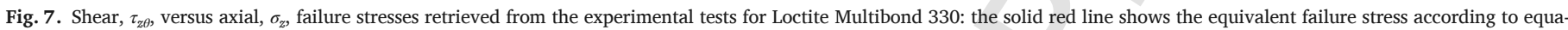

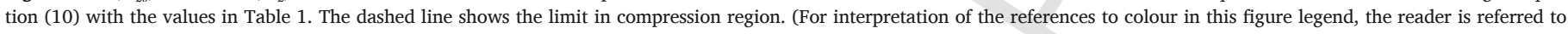
the Web version of this article.)

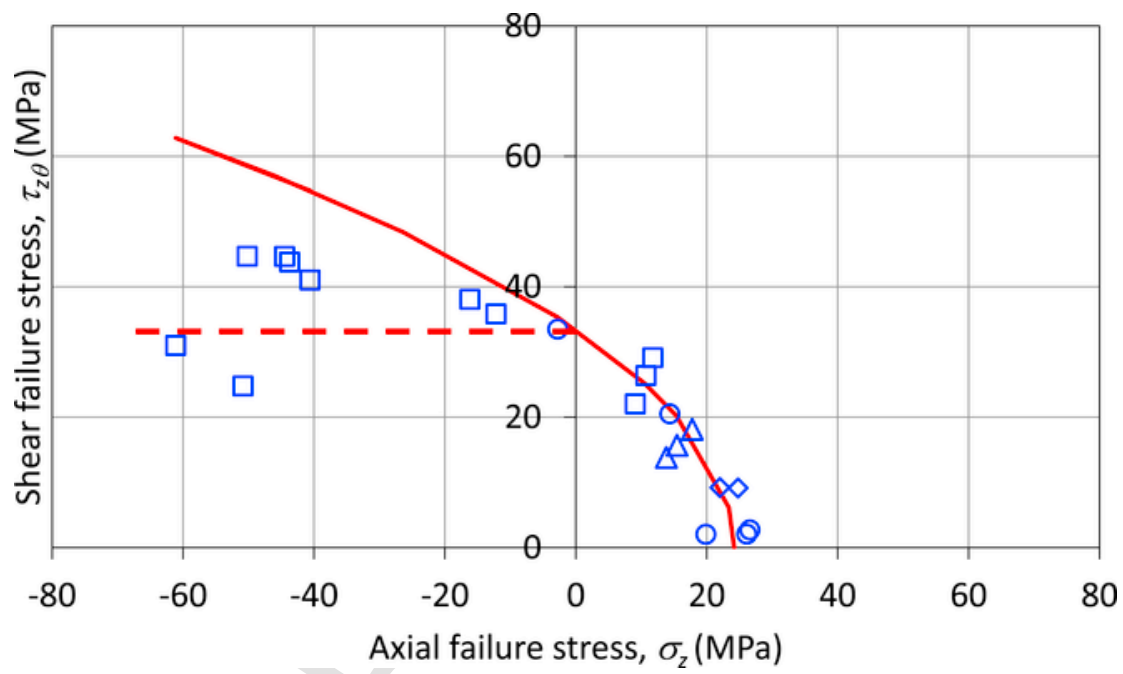

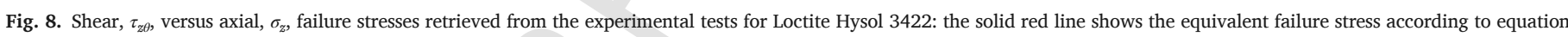

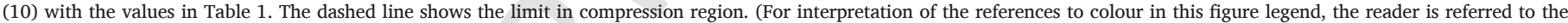
Web version of this article.)

Table 2

Definition of the proposed criterion.

\begin{tabular}{ll}
\hline Applied stress & Failure criterion \\
\hline$\sigma_{\text {peel }}<0, \forall \tau$ & $\tau \leq \tau_{c r}$ \\
$\sigma_{\text {peel }} \geq 0, \forall \tau$ & $\tau^{2}+A \sigma \leq \tau_{c r}^{2}$ \\
\hline
\end{tabular}

plying the adhesives mainly to avoid any fillet or leakage of adhesive in the groove, which could modify the stiffness of the system creating stress concentrations. Therefore, we applied a PTFE insert inside the tubular specimen in the bottom adherend, designed to have a little interference. This PTFE pin prevents the adhesive to leak inside the joint, where it would have been hard to be removed and ensures the concentricity between the upper and lower adherends. We did not use a system to enforce the adhesive thickness, such as calibrated glass spheres or other micro-shims, since we do not want to introduce stress concentrations in the adhesive, thus we pressed together the adherends, by applying a $0.2 \mathrm{~kg}$ dead weight on top one, until the adhesive in excess is squeezed out externally. This procedure was carried out for both ad- hesives, the only difference consists in the application of the activator on one adherend for the Multibond 330. After having assembled the joint, we removed the adhesive from the grooves with a knife and then we started the curing procedure. The curing time is very different for the two adhesives (very fast for the 3422, slow for the 330), but we decided to let the specimens cure at room temperature for $72 \mathrm{~h} \mathrm{~h}$ in order to ensure the complete strength for both. After the curing, we easily removed the central pin from the tubular joint thanks to the non-stick properties of the PTFE.

The experimental set up is reported in Fig. 4. The specimen is coupled to the electro-hydraulic testing machine MTS MINI Bionix 858, equipped with a biaxial load cell ( $25 \mathrm{kN}$ axial and $200 \mathrm{Nm}$ torsional). Since the hydraulic clamps installed on the testing machine are not sufficient to grip the specimen directly, we used two high strength steel pins to transfer the load and the torque to the joint.

The FlexTest software was programmed to control simultaneously the axial load and the applied torque, both applied quasi statically, in order to limit possible dynamic and viscoelastic effects. In order to enforce the prescribed loading conditions described by Fig. 3, we programmed a fixed axial load rate at $100 \mathrm{~N} / \mathrm{min}$ for all the loading condi- 


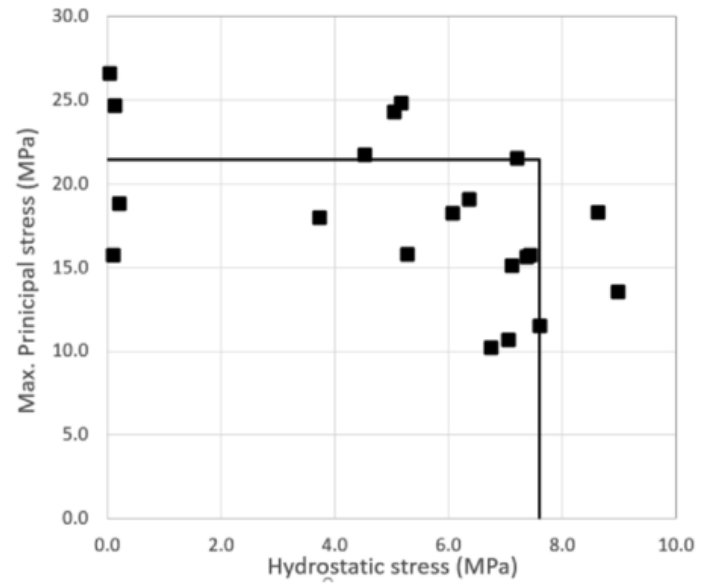

(a)

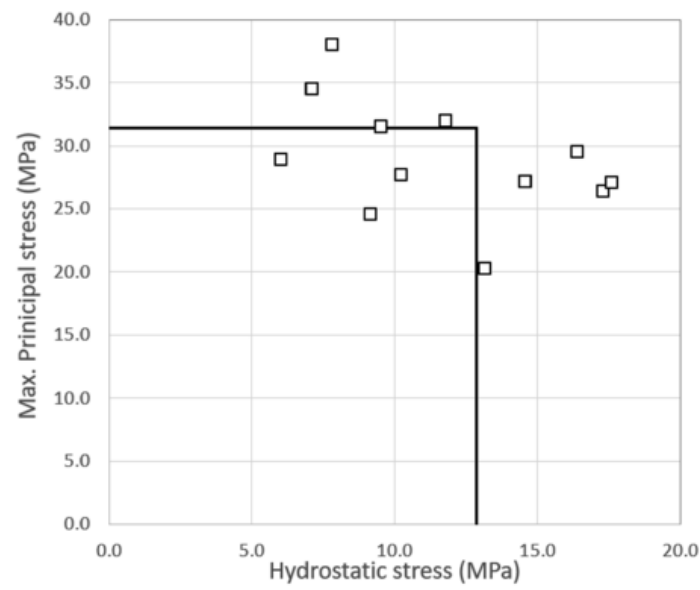

(b)

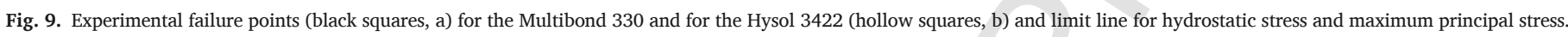

Table 3

LHS + LMPS criterion parameters.

\begin{tabular}{lll}
\hline Adhesive & LHS_cr [MPa] & LMPS_cr [MPa] \\
\hline Loctite Multibond 330 & 7.6 & 21.3 \\
Loctite Hysol 3422 & 12.9 & 31.4 \\
\hline
\end{tabular}

tions and we computed the correspondent shear rate to enforce the desired configuration. For each configuration, three replicates were performed in order to have a statistically reliable set of data. We investigated two different adhesives: Loctite Multibond 330, which is a general purpose acrylic adhesive [37], and Loctite Hysol 3422, a two component, fast curing, epoxy resin [38]. We considered a Poisson's ratio of 0.33 for both adhesives. This two adhesives exhibit a quite brittle [20] [39], behaviour, so the application of a simple elastic stress analysis could be adopted. Typical applications for these adhesives are structural bonding with good stiffness and good gap filling properties, typical for metallic parts.

\subsection{Stress analysis}

Thanks to the simple geometry adopted, it is possible to calculate analytically the stress components from the axial and torsional load applied to the joint. Considering a cylindrical coordinate system $(r, \theta, z)$, the axial and shear stress components on an infinitesimal cubic element of the adhesive can be written as:

$\sigma_{z}=\frac{F}{\pi\left(r_{e}^{2}-r_{i}^{2}\right)}$

$\tau_{z \theta}=\frac{M_{t}}{\frac{\pi\left(r_{e}^{4}-r_{i}^{4}\right)}{2}} r_{e}$

where $F$ is the axial force, $M_{t}$ the applied torque, $r_{e}$ and $r_{i}$ are the outer and inner radius of the tubular joint. Since the outer and inner radius of the tubular joint $r_{e}$ and $r_{i}$ are $15 \mathrm{~mm}$ and $10 \mathrm{~mm}$ respectively, the shear stress, which has a linear distribution depending on the radius, does not vary much inside the adhesive layer. In addition, the radial and circumferential constraints applied to the compliant adhesive layer by the stiff steel adherends, induce two indirect stress components, which are promoted by Poisson's effect. According to generalized Hooke's law it is possible to write:
$\varepsilon_{r}=\frac{1}{E}\left(\sigma_{r}-v\left(\sigma_{\theta}+\sigma_{z}\right)\right)=0$

$\varepsilon_{\theta}=\frac{1}{E}\left(\sigma_{\theta}-v\left(\sigma_{r}+\sigma_{z}\right)\right)=0$

where $\varepsilon_{r}$ is the radial strain, $\varepsilon_{\theta}$ is the circumferential strain, $E$ is the adhesive Young's modulus, $\sigma_{r}$ and $\sigma_{\theta}$ are the radial and hoop stress components, and $\nu$ is the Poisson's ratio. Simultaneous solution of (4) and (5) gives the radial and circumferential stresses as:

$\sigma_{r}=\sigma_{\theta}=\frac{v}{1-v} \sigma_{z}$

The occurrence of these secondary stress components (radial and circumferential) proves that this specimen generates in the thin adhesive film the same triaxial stress state typically occurring in bonded joints, which is particularly critical for adhesives. To investigate a failure criterion for the adhesive, the principal stress components were retrieved as the eigenvalues of the stress tensor, and can be written as:

$\sigma_{1}=\frac{\sigma_{z}+\sqrt{\sigma_{z}^{2}(1-2 v)^{2}+4 \tau_{z \theta}^{2}(1-v)^{2}}}{2(1-v)}$

$\sigma_{2}=\frac{v}{1-v} \sigma_{z}$

$\sigma_{3}=\frac{\sigma_{z}-\sqrt{\sigma_{z}^{2}(1-2 v)^{2}+4 \tau_{z \theta}^{2}(1-v)^{2}}}{2(1-v)}$

\section{Results and discussion}

Fig. 5 shows the results obtained from the experimental tests in terms of shear, $\tau_{z \theta}$, versus axial, $\sigma_{z}$, failure stresses for the Loctite Multibond 330 adhesive with solid dots and displays the results for the Loctite Hysol 3422 adhesive with hollow dots.

These diagrams highlight that

- for both adhesives, the replications of the tests provide repeatable results, in particular when tensile stresses are applied (Fig. 5);

- both adhesives show a quite similar trend in terms of shear failure stress versus axial failure stress (Fig. 5); 


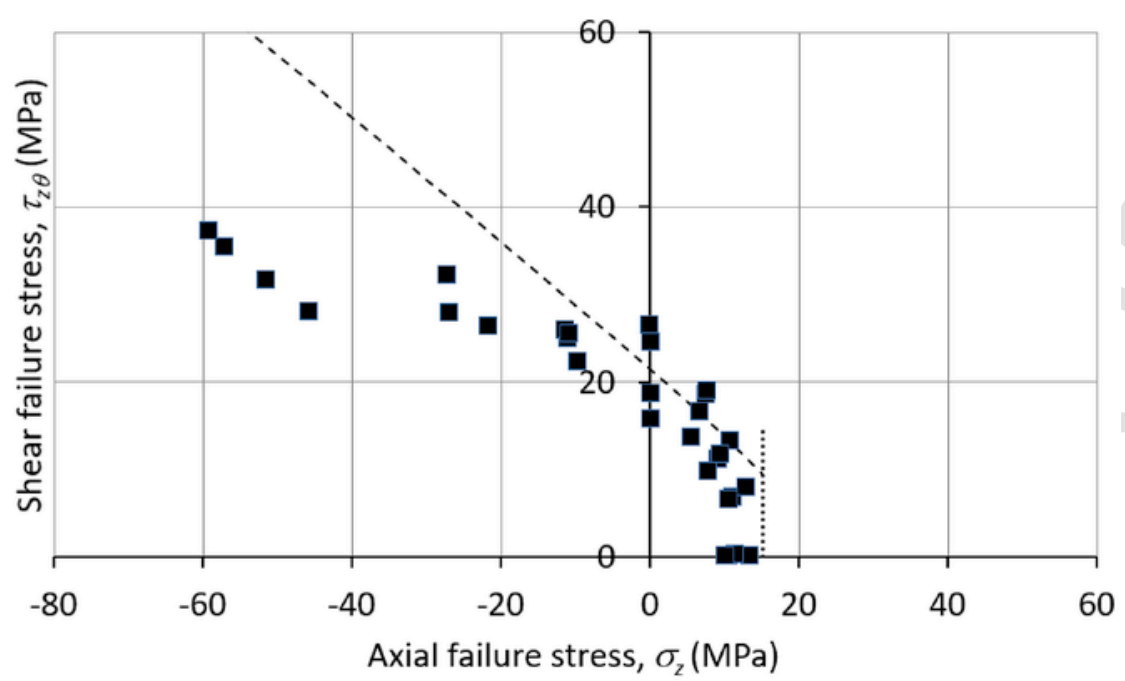

(a)

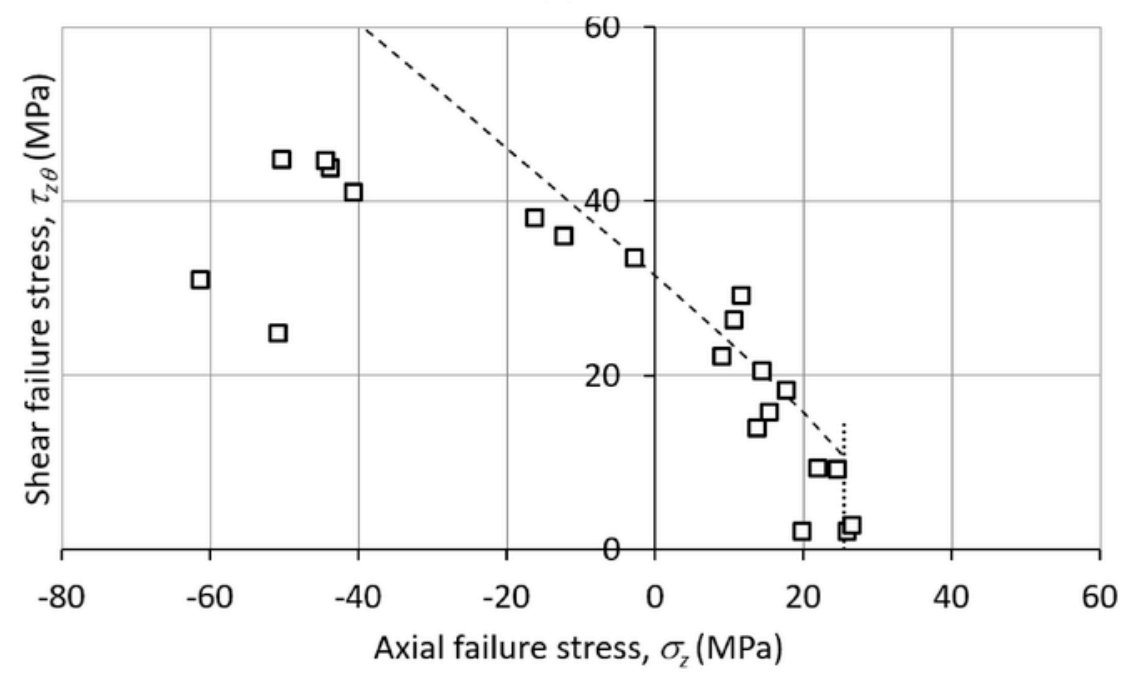

(b)

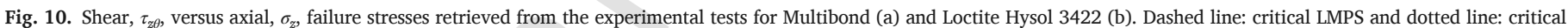
LHS.

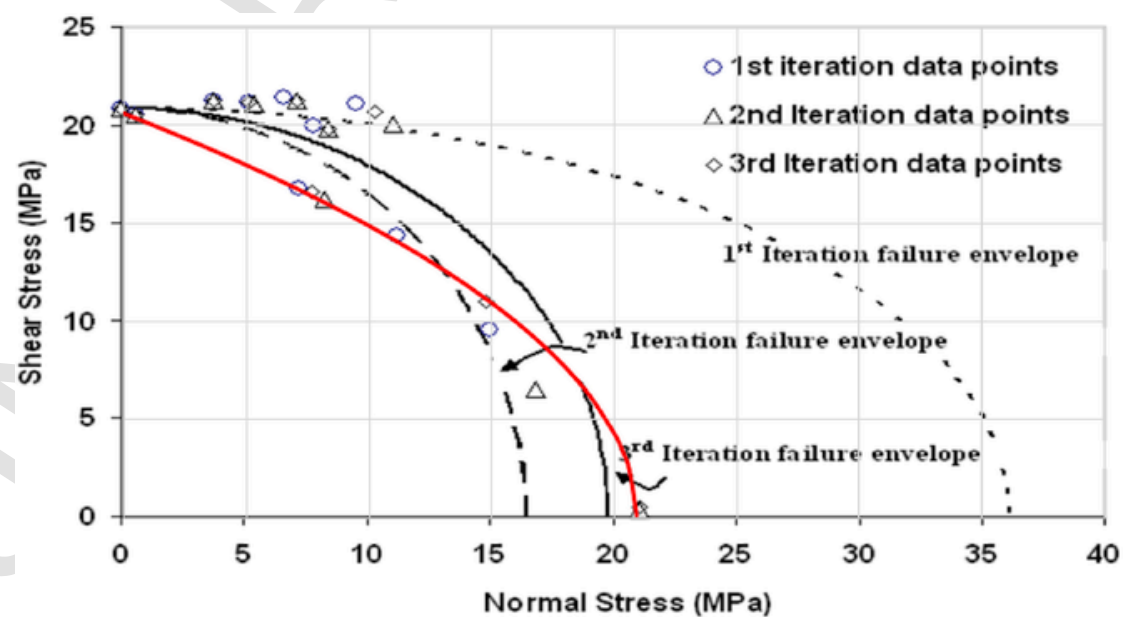

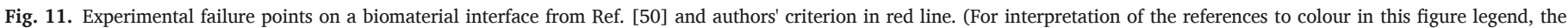
reader is referred to the Web version of this article.) 


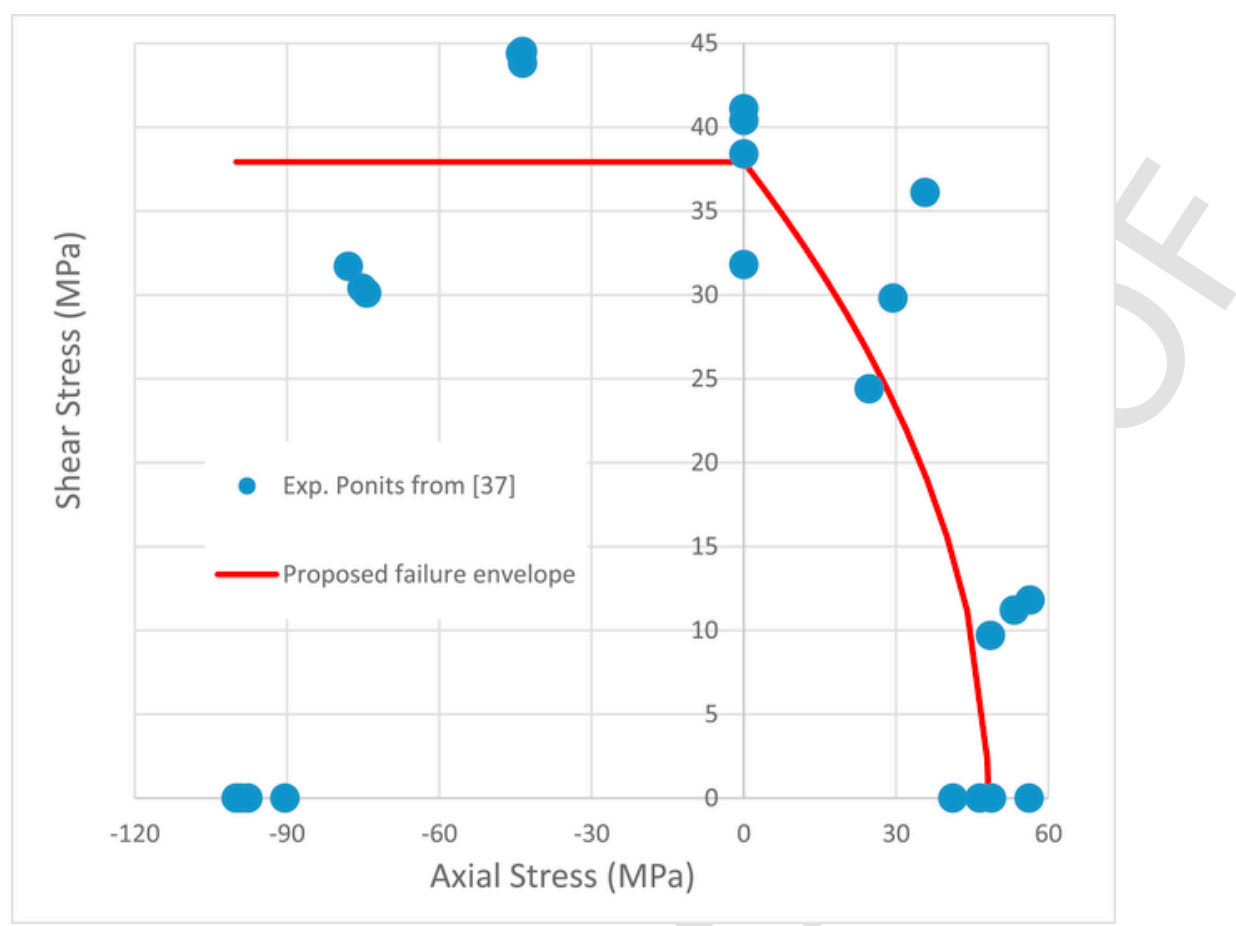

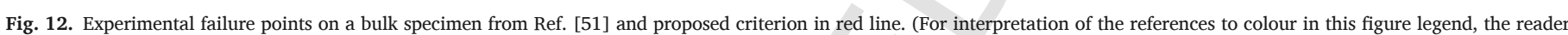
is referred to the Web version of this article.)

- on the whole range here examined, the Loctite Hysol 3422 adhesive exhibits a significantly higher strength than the Loctite Multibond 330 (Fig. 5).

Fig. 6 shows the equivalent stress according to Von Mises, maximum principal stress or Rankine criterion and Stassi d'Alia [40] failure criteria as a function of the stress triaxiality index. It appears that all the examined equivalent stresses remarkably depend on the stress triaxiality index, without any clear relationship. Thus, the classical equivalent stress theories (i.e Tresca or von Mises equivalent stress) cannot be applied to these type of adhesives, as already suggested in Refs. [41], [42]. The Stassi d'Alia criterion, which is lesser known, deserves a further explanation. The idea is to link the equivalent stress to two simple quantities, the uniaxial tensile stress, $\sigma_{0}$ and the uniaxial compressive stress, $\sigma_{0}^{\prime}$. The ratio $\rho=\frac{\sigma_{0}^{\prime}}{\sigma_{0}}$ is used to define the equivalent stress $\sigma_{\text {eq_SDA }}$ using the following formula, which limits the deformation energy and takes into account the effect of the first invariant as well:

$\rho \sigma_{e q \_S D A}^{2}-(\rho-1)\left(\sigma_{1}+\sigma_{2}+\sigma_{3}\right) \sigma_{e q \_S D A}-\left[\left(\sigma_{1}^{2}+\sigma_{2}^{2}+\sigma_{3}^{2}\right)-\left(\sigma_{1} \sigma_{2}+\sigma_{2} \sigma_{3}\right.\right.$. $\rho \sigma_{e q \_S D A}^{2}-(\rho-1)\left(\sigma_{1}+\sigma_{2}+\sigma_{3}\right) \sigma_{e q \_} S D A-\left[\left(\sigma_{1}^{2}+\sigma_{2}^{2}+\sigma_{3}^{2}\right)-\left(\sigma_{1} \sigma_{2}+\sigma_{2} \sigma_{3}\right.\right.$.

This criterion behaves better than the other two, according to Fig. 6 since the equivalent stress is less affected by the stress triaxiality and therefore it is possible to envision a constant failure stress limit, especially for the Multibond 330.

Among the stress criteria retrievable in the literature, the combination of peak of the Local Hydrostatic Stress (LHS) and the peak of Local Maximum Principal Stress (LMPS) as suggested by Carraro and Quaresimin [43] can be quite successfully applied to the experimental data. Their work suggests that the first one is suitable when there is little or no shear stress, while the last one hold in case of predominant shear. We show a comparison of the proposed criterion and the LHS + LMPS in the Discussion section.

\section{Proposed failure criterion}

Many failure criteria are proposed in technical literature for bonded joints [44]- [47], and most of them rely on fracture toughness and fracture mechanics. The applicability of these criteria in the industrial world is limited due to the complexity of the experimental tests needed to obtain reliable data about the adhesives. Therefore, the authors propose a simple stress-based failure criterion which needs only monoaxial properties retrievable from standard experimental tests. The critical failure stress in the adhesive joint is given by the following relationship:

$\tau^{2}+A \sigma \leq \tau_{c r}^{2}$

where $\tau$ and $\sigma$ are respectively the applied shear and the axial stress, $A$ is a coefficient to be identified from the experimental tests, and finally, $\tau_{c r}$ is the critical failure shear stress.

By assuming the failure shear stress, $\tau_{c r}$, as the average value retrieved experimentally in pure shear mode, and calculating the parameter $A$ in the pure tensile stress case, i.e. $A=\tau_{c r}^{2} / \sigma_{a v}$ (where $\sigma_{a v}$ is the average pure tensile failure value retrieved experimentally), the experimental data from the adhesives yield the values reported in Table 1.

One important point to be highlighted is that the failure criterion proposed is intended for the incipient failure of the entire joint, while no assumption about the plasticity of adhesive are considered. Even though nowadays the modern adhesives are strong and tough and allow plastic deformation it is still preferable, as a design rule, to stay within their elastic limit, leaving the plastic reserve to prevent catastrophic failure. Considering only the elastic part is a strong simplification, but, since the aim is to provide a simple tool for an industrial designer to estimate and predict the failure load of bonded joints under multiaxial stresses it seems a quite simple and conservative approach also for ductile adhesives.

Fig. 7 shows the experimental data from the Loctite Multibond 330 adhesive with the failure stress (solid red curve) according to equation 
(10). Similarly, Fig. 8 shows the experimental data from the Loctite Hysol 3422 with the failure stress (solid red curve) according to equation (10).

These diagrams highlight that the proposed failure criterion accurately describes the response of the adhesive for positive axial stresses. By contrast, in case of compressive stresses, the failure stress prediction is significantly higher than the experimental value. For both adhesives, when the mixed mode stress state become compressive, the pure shear strength can be assumed as the critical value. Since the proposed methods relies only on the pure tensile and pure shear experimental characterization of the adhesive in thin film, this appears as a very simple but fairly accurate failure criterion. The main advantage in exploiting this kind of criterion is that, by using data coming from pure tensile test and pure shear test it is possible to have a simple but accurate prediction of the mixed mode behaviour. The proposed criterion is less accurate when shear and compression are applied together, probably due to the very low thickness of adhesive, which makes it very difficult to achieve a true compression failure.

Therefore, a simple conservative criterion can be envisioned. Given the results of pure tensile test and pure shear test obtained experimentally, we have the following equivalent stress in mixed mode. If the normal stress is positive the critical equivalent stress is given by equation (10), with parameter $A$ determined by $A=\tau_{c r}{ }^{2} / \sigma_{a v}$. If the normal stress is negative the critical equivalent stress is equal to the failure stress under pure shear, represented by the red dashed line in Figs. 7 and 8. Table 2 shows the definition of the proposed criterion, where the two unknown parameters, $A$ and $\tau_{c r}$ must be found from uniaxial experimental test.

Another possible criterion which fits the experimental results at least in the traction-torsion region is the one proposed by the group of Prof. Quaresimin [43] [48], [49]. In general, what both approaches have in common is a simple way to find out a criterion by using only the information given by a simple mechanical test, both in pure shear and pure tension [43], which is easier to carry out compared to other mixed mode tests. In order to apply this method, it is convenient to report the experimental failure points in the hydrostatic - maximum principal stress plane (Fig. 9). Once the points are displayed in this plane it is possible to envision a rectangular failure region and limit for the two stress component being considered, which can be computed starting from the same information of Table 1, the pure shear and the pure tensile load, which are used to compute the hydrostatic stress and the maximum principal stress. We reported the limit stress in Table 3, for both adhesives. Once the limit critical Limit Hydrostatic Stress (LHS) and Limit Maximum Principal Stress (LMPS) are found we can easily depict in the canonical $\sigma-\tau$ plane the curves obtained by imposing these limits, as shown in Fig. 10. By simply comparing the Carraro's failure locus and the proposed failure region, it is simple to envision that the authors' failure criterion is more conservative, since it does not consider any positive contribution of the compressive state, as testified by the horizontal line in the shear-compression quadrant. Conversely, the Carraro's criterion presents a situation which is typical of many stress based criteria, i.e. the larger the compressive stress, the higher the critical stress. This behaviour may be good for some kind of adhesives, which are particularly sensitive to the hydrostatic stress level, but in general is less conservative. Besides, many of the experimental points in the compressive region do not fall inside the safety region proposed.

\subsection{Application of the proposed criterion to literature data}

Considering the work of Chowdhuri and Xia [50] it is possible to apply the proposed criterion to another set of experimental points, retrieved on different experimental set-up. This work considers a biomaterial spherical interface between an epoxy resin and aluminum. The geometry is very different from the one considered to develop the failure criterion, the stresses are not uniform, but not singular and therefore this comparison is very challenging for the criterion. The data are retrieved from the article [50] and elaborated to estimate the new values for the proposed criterion.

The application of the criterion lead to:

$\tau_{c r_{\text {exp }}}=20.6 \mathrm{MPa}$

$\sigma_{a v \exp }=20.9 \mathrm{MPa}$

$A=\frac{\bar{\tau}_{c r \_ \text {exp }}^{2}}{\sigma_{\text {av_exp }}}=20.3 \mathrm{MPa}$

The failure envelope is therefore easily calculated using the new set of data and Table 2 equations and superimposed to the experimental points in Fig. 11. The failure envelope computed with the proposed criterion is reported in red, while the experimental points are the dots. The result shows a good agreement between the data and the envelope, which is conservative in case of low axial stresses and more precise for higher axial stresses.

Another challenging test bench for the proposed method is its application to the work of Zarouchas et al. [51], which is based on a bulk test specimen of EPIKOTETM MGS Paste 135/G. Since the method was developed for adhesive in thin layers, we expect a different stress triaxiality contribution in this bulk test, since there is no effect of the adherends. This difference could be important in the failure prediction. It is possible to report the experimental failure points obtained by Ref. [51] and compare it with the failure criterion of Table 2. The results are shown in Fig. 12, where it is clear that the bulk test is suitable to be predicted with the proposed failure criterion only where the axial stress is non-negative. The prediction in the right quadrant is quite good, the error is due to the normal experimental deviation of adhesive tests. The proposed failure envelope in the left quadrant can be good only for moderate low axial stress, but when the compression becomes too predominant other failure modes arises and the criterion, developed for thin adhesive layers, cannot be used anymore. It is interesting that the authors in Ref. [51] finds out that the Stassi d'Alia criterion fits very good the experimental points, as for the set of experimental points in the present work. The applicability of Stassi d'Alia criterion depends on its low sensitivity to the triaxiality index, and it is thus useful to predict a correct failure envelope.

\section{Conclusions}

The present work deals with the problem of estimating the strength of structural adhesive in thin films, with the purpose to find a simple stress criterion applicable in general industrial problems, with low experimental efforts and limited numerical resources. The experimental tests are carried out by exploiting a tubular butt bonded specimen with relieve grooves, used to achieve a regular stress distribution inside the adhesive layer. The stress analysis emphasizes that all the stress components are present, due to Poisson's effect and the stress triaxiality playing a major role. We tested two chemically different adhesives and analyzed the results in the Mohr plane to evaluate several literature criteria. The results confirm that traditional stress approaches do not lead to good predictions, since the hydrostatic stress is not taken into account. By following the authors' proposal, it is possible to combine the data obtained in pure tensile test and pure shear test, to predict the mixed mode behaviour with good agreement with the experimental data. The adhesive mechanical behaviour, when constrained by the adherends, seems to be quite insensitive to compressive stresses and the proposed criterion considers this peculiar aspect. The main finding of this work is that, by using the provided criterion, it is possible to design an adhesive bonded joint under a combination of multiaxial stresses, knowing the adhesive behaviour in pure shear and in pure tension only. 


\section{Uncited references}

[14] [15]; [18] [45]; [46].

\section{References}

[1] G. Dolev, Mechanical characterization of adhesive layer in-situ and as bulk material, J Adhes 12 (1981) 283-294.

[2] R.D. Adams, J. Comyn, W.C. Wake, Structural adhesive joints in engineering, In: Struct. adhes. Joints eng, Chapman and Hall, 1997.

[3] J.M. Arenas, J.J. Narbón, C. Alía, Optimum adhesive thickness in structural adhesives joints using statistical techniques based on Weibull distribution, Int J Adhesion Adhes 30 (3) (Apr. 2010) 160-165.

[4] D. Gleich, M. Van Tooren, Analysis and evaluation of bondline thickness effects on failure load in adhesively bonded structures, J Adhes Sci Technol 15 (9) (2001) 1091-1101.

[5] Araldite ${ }^{\circledR}$ rapid product data property, 2011, [Online]. Available: www. go-araldite.com, Accessed 23 July 2018.

[6] Loctite, Design guide for bonding plastics, 6 (800) (2011).

[7] M. Goland, E. Reissner, The stresses in cemented joints, J Appl Mech 11 (1944) A17-A27.

[8] P. Chalkley, F. Rose, Stress analysis of double-strap bonded joints using a variational method, Int J Adhesion Adhes 21 (3) (Jan. 2001) 241-247.

[9] H.L. Groth, Stress singularities and fracture at interface corners in bonded joints, Int J Adhesion Adhes 8 (2) (Apr. 1988) 107-113.

[10] G.P. Anderson, S.J. Bennett, K.L. Devries, Analysis and testing of adhesive bonds, Academic Press, Inc., New York, 1977.

[11] D. Morin, G. Haugou, B. Bennani, F. Lauro, Identification of a new failure criterio for toughened epoxy adhesive, Eng Fract Mech 77 (17) (Nov. 2010) 3481-3500.

[12] L.F.M. Da Silva, R.D. Adams, M. Gibbs, Manufacture of adhesive joints and bulk specimens with high-temperature adhesives, Int J Adhesion Adhes 24 (2004) 69-83.

[13] A. Adnan, C.T. Sun, Effect of adhesive thickness on joint strength: a molecular dynamics perspective, J Adhes 84 (5) (Jun. 2008) 401-420.

[14] M. Fischer, M. Pasquier, Shear behaviour of structural adhesives in the bondline, Construct Build Mater 3 (1) (Mar. 1989) 31-34.

[15] M. Imanaka, A. Fujinami, Y. Suzuki, Fracture and yield behavior of adhesively bonded joints under triaxial stress conditions, J Mater Sci 5 (2000) 2481-2491.

[16] M. Ignjatovic, P. Chalkley, C. Wang, The yield behaviour of a structural adhesive under complex loading, 1998.

[17] A. Spaggiari, E. Dragoni, Effect of mechanical surface treatment on the static strength of adhesive lap joints, J Adhes 89 (9) (Sep. 2013) 677-696.

[18] G.W. Critchlow, K.A. Yendall, D. Bahrani, A. Quinn, F. Andrews, Strategies for the replacement of chromic acid anodising for the structural bonding of aluminium alloys, Int J Adhesion Adhes 26 (6) (Sep. 2006) 419-453.

[19] D. Castagnetti, A. Spaggiari, E. Dragoni, Effect of bondline thickness on the static strength of structural adhesives under nearly-homogeneous shear stresses, J Adhes 87 (7-8) (2011) 780-803.

[20] E. Dragoni, L. Goglio, F. Kleiner, Designing bonded joints by means of the JointCalc software, Int J Adhesion Adhes 30 (5) (Jul. 2010) 267-280.

[21] L. Goglio, M. Rossetto, Evaluation of the singular stresses in adhesive joints, J Adhes Sci Technol 23 (10-11) (Jan. 2009) 1441-1457.

[22] L. Goglio, M. Rossetto, Stress intensity factor in bonded joints: influence of the geometry, Int J Adhesion Adhes 30 (5) (Jul. 2010) 313-321.

[23] R.D. ADAMS, N.A. PEPPIATT, Stress analysis of adhesive-bonded lap joints, J Strain Anal Eng Des 9 (3) (1974) 185-196.

[24] J.J.T. Tomblin, W. Seneviratne, H. Kim, J. Lee, Characterization of in-plane, shear-loaded adhesive lap joints: experiments and analysis, May, 2003, FAA rep. DOT/FAA/AR-03/21.

[25] D. Castagnetti, A. Spaggiari, E. Dragoni, Robust shape optimization of tubular butt joints for characterizing thin adhesive layers under uniform normal and shear stresses, J Adhes Sci Technol 24 (11-12) (Jan. 2010) 1959-1976.
[26] J.L. Högberg, U. Stigh, Specimen proposals for mixed mode testing of adhesive layer, Eng Fract Mech 73 (16) (Nov. 2006) 2541-2556.

[27] Y.-J. Guo, Y.J. Weitsman, A modified specimen for evaluating the mixed mode fracture toughness of adhesives, Int J Fract 107 (3) (2001) 201-234.

[28] G. Fernlund, J.K. Spelt, Mixed-mode fracture characterization of adhesive joints, Compos Sci Technol 50 (4) (Jan. 1994) 441-449.

[29] L. Alfonso, C. Badulescu, N. Carrere, Use of the modified Arcan fixture to study the strength of bonded assemblies for automotive applications, Int J Adhesion Adhes 80 (Jan. 2018) 104-114.

[30] J.M. Greer, S.E. Galyon Dorman, M.J. Hammond, Some comments on the Arcan mixed-mode (I/II) test specimen, Eng Fract Mech 78 (9) (Jun. 2011) 2088-2094.

[31] Henkel, "LOCTITE ${ }^{\circledR} 330^{\mathrm{TM}}$," 2009. [Online]. Available: www.loctite.com. [Accessed: 23-01-2018].

[32] Henkel, Hysol ${ }^{\circledR} 3422$ PRODUCT DESCRIPTION, 2003, [Online]. Available: https: //docs-emea.rs-online.com/webdocs/0606/0900766b806067cd.pdf Accessed 23 January 2018.

[33] D.C. Montgomery, Design and analysis of experiments, In: Des. anal. exp, John Wiley and Sons, 2004.

[34] F. Chen, S. Gatea, H. Ou, B. Lu, H. Long, Fracture characteristics of PEEK at various stress triaxialities, J. Mech. Behav. Biomed. Mater. 64 (Dec. 2016) 173-186.

[35] S.G.G. Prolongo, a. Ureña, "Effect of surface pre-treatment on the adhesive strength of epoxy-aluminium joints, Int J Adhesion Adhes 29 (1) (Jan. 2009) 23-31.

[36] L.F.M.M. da Silva, R.J.C.J.C. Carbas, G.W.W. Critchlow, M. a. V. Figueiredo, K. Brown, Effect of material, geometry, surface treatment and environment on the shear strength of single lap joints, Int J Adhesion Adhes 29 (6) (Sep. 2009) 621-632.

[37] Loctite multibond 330 TdS." [Online]. Available: https://tds.us.henkel.com/NA/ UT/HNAUTTDS.nsf/web/65B0DB8566343883882571870000D66C/\$File/ AA330-EN.pdf. [Accessed: 09-01-2015].

[38] Loctite hysol 3422." [Online]. Available: https://www.kaindltech.at/fileadmin/ Datenblaetter/Datenblaetter/Loctite/3422-EN.pdf. [Accessed: 09-01-2015].

[39] R. Carbas, L. da Silva, G. Critchlow, Effect of post-cure on adhesively bonded functionally graded joints by induction heating, Proc Inst Mech Eng Part L J. Mater. Des. Appl. 229 (5) (Oct. 2015) 419-430.

[40] A. Spaggiari, D. Castagnetti, E. Dragoni, Mixed-mode strength of thin adhesive films: experimental characterization through a tubular specimen with reduced edge effect, J Adhes 89 (8) (Aug. 2013) 660-675.

[41] W.R. Broughton, L.E. Crocker, Project MMS11 report 4 assessment of predictive analysis for bonded and bolted t-joints, In: Solutions, May, 2004.

[42] W.R. Broughton, L.E. Crocker, J.M. Urquhart, Strength of adhesive joints: A parametric study, 2001.

[43] P.A. Carraro, M. Quaresimin, A damage based model for crack initiation in unidi rectional composites under multiaxial cyclic loading, Compos Sci Technol 99 (2014) 154-163.

[44] A.D. Crocombe, D.A. Bigwood, G. Richardson, Analysing structural adhesive joints for failure, Int J Adhesion Adhes 10 (3) (1990) 167-178.

[45] P.H. Martiny, F. Lani, a. J. Kinloch, T. Pardoen, A maximum stress at a distance criterion for the prediction of crack propagation in adhesively-bonded joints, Eng Fract Mech 97 (1) (2012) 105-135.

[46] P. Weißgraeber, W. Becker, Finite Fracture Mechanics model for mixed mode fracture in adhesive joints, Int J Solid Struct 50 (14-15) (2013) 2383-2394.

[47] S. Hell, P. Weißgraeber, J. Felger, W. Becker, A coupled stress and energy criterion for the assessment of crack initiation in single lap joints: a numerical approach, Eng Fract Mech 117 (2014) 112-126.

[48] M. Quaresimin, A damage-based approach for the fatigue design of composite structures, IOP Conf Ser Mater Sci Eng 139 (2016) 012006.

[49] P.A. Carraro, L. Maragoni, M. Quaresimin, Prediction of the crack density evolution in multidirectional laminates under fatigue loadings, Compos Sci Technol 145 (145) (Jun. 2017) 24-39.

[50] M.A.K. Chowdhuri, X. Zihui, An innovative method to determine bonding strength envelope based on theory of Bi-Material interface mechanics, Procedia Eng. 10 (2011) 118-123.

[51] D. Zarouchas, R. Nijssen, Mechanical behaviour of thick structural adhesives in wind turbine blades under multi-axial loading, J Adhes Sci Technol 30 (13) (Jul. 2016) 1413-1429. 